\title{
IAMJ
}

INTERNATIONAL

AYURVEDIC

MEDICAL JOURNAL

Review Article

ISSN: 23205091

Impact Factor: 5.344

\section{A REVIEW ON LAAKSHA HARIDRADI DHOOPA}

Ravikrishna. $\mathbf{S}^{1}$, Sreejith. $\mathbf{K}^{2}$

${ }^{1}$ Associate Professor, ${ }^{2}$ Assistant Professor,

Department of Agadatantra, Sri Dharmasthala Manjunatheshwara College of Ayurveda and Hospital, Kuthpady, Udupi-574118, Karnataka, India

Corresponding Author: sreevaipur@gmail.com

https://doi.org/10.46607/iamj09p4052020

(Published online: July 2020)

Open Access

(C) International Ayurvedic Medical Journal, India 2020

Article Received: 27/06/2020 - Peer Reviewed: 04/07/2020 - Accepted for Publication: 07/07/2020

Check for updates

\section{ABSTRACT}

Visha chikitsa is one among the ashtanga Ayurveda which primarily focuses on the prognosis, diagnosis and management of diseases caused by the Visha Dravyas, either Sthavara (plant origin), Jangama (animal origin) or kritrima(artificial poison). In this modern era, due to urbanization \& industrialization, pollution possesses a major threat to the mankind and other biotic components of the ecosystem. This paves the way for imbalance in harmony of the ecosystem thereby leading to various health hazards such as severe respiratory illness and other diseases.

There are many fruitful agada Yogas which are mentioned in our classics. Laaksha haridradi dhoopa is one such formulation mentioned in Susrutha Samhita Kalpasthana, 3rd chapter Jangama Visha Vijnaniya Adhyaya. Dhoopana karma is one of the traditional practices which uses the anti-bacterial activity of the smoke produced by the drugs. This yoga comprises of ten such vishagna dravyas.

Keywords: Visha, Dhoopana, Agada

\section{INTRODUCTION}

Dhoopana literally means fumigation and is one of the methods that have been widely used in the ancient era as well as in the present scenario for the protection from various microbes. Our classics had detailed explanation of different types of microbes, the diseases caused by them and their treatment modalities. ${ }^{1}$ Among the different modalities one is Dhoopana Karma. Here the role of fumigation comes into action. The Dhoopana is prepared out of Jangama (animal origin-hair, nails etc.) and Sthavara Dravyas (plant origin- Haridra, Guggulu 
etc.) which possess medicinal as well as anti-microbial properties. In ancient era, different techniques like Yaga, Homa, Havana has been performed to make the vicinity free of microbes. The usage of these methods emphasis its significant role in the reduction of microbial levels.

In the present scenario, due to globalisation and industrialization one of the challenging problems that the entire world faces is pollution. These pave the way for emergence of different epidemics which has its drastic effect on biotic systems. To some extent the extensive use of these Dhoopana Dravyas with its multidimensional facets will play a leading role in controlling the microbial load thereby resulting in significant decrease in the pollution and its harmful effects.
Laaksha Haridradi Dhoopa is a preparation mentioned in Sushruta Samhita Kalpasthana third chapter ${ }^{2}$. Where he explained the features of polluted air like the birds get exhausted and fall on ground, the human being will suffer from diseases like Kasa, Pratishyaya, Shiroruja, Nayanamaya and its treatment by using this formulation.

\section{Materials and Methods:}

For the present review study, the primary sources of literatures are the Ayurvedic textbooks and relevant modern textbooks.

\section{Review of literature:}

This formulation explained by Acharya Susrutha in Kalpasthana 3rd chapter sloka no.17 comprises of drugs which are elaborated in table no.1

Table 1: Ingredients of Laaksha haridradi dhoopa with Botanical identity

\begin{tabular}{|l|l|l|l|}
\hline S. No & Drug Name & Botanical name & Family \\
\hline 1 & Laksha & Laccifer lacca (Kerr) & Coccideae \\
\hline 2 & Haridra & Curcuma longa L. & Zingiberaceae \\
\hline 3 & Ativisha & Aconitum heterophyllum Wall.ex Royle. & Ranunculaceae \\
\hline 4 & Abhaya & Terminalia chebula Retz. & Combretaceaea \\
\hline 5 & Abdha & Cyperus rotundus L. & Cyperaceae \\
\hline 6 & Harenuka & Vitex negundo L. & Lamiaceae \\
\hline 7 & Ela & Elettaria cardamomum Maton. & Zingiberaceae \\
\hline 8 & Vakra & Valeriana wallichii DC. & Valerianaceae \\
\hline 9 & Kushta & Saussurea lapa (Decne)Sch.Bip & Asteraceae \\
\hline 10 & Priyangu & Callicarpa macrophylla Vahl. & Verbenaceae \\
\hline
\end{tabular}

Table 2: Ingredients of Laaksha haridradi dhoopa and Their Properties

\begin{tabular}{|c|c|c|c|c|c|c|c|}
\hline S.No & Drug Name & Rasa & Guna & Virya & Vipaka & Karma & Gana \\
\hline 1 & Laksha ${ }^{3}$ & Tikta, Kashaya & $\begin{array}{l}\text { Laghu, } \\
\text { Snigdha }\end{array}$ & $\begin{array}{l}\text { Ushna- } \\
\text { Anushna }\end{array}$ & Katu & $\begin{array}{l}\text { Sandhaniya, Stambhana, } \\
\text { Varnya, } \\
\text { Balya, } \\
\text { Kushtagna, Vrunaro- } \\
\text { pana, } \\
\text { Kasaghna }\end{array}$ & Lakshadi Varga \\
\hline 2 & Haridra $^{4}$ & $\begin{array}{l}\text { Tikta, } \\
\text { Katu }\end{array}$ & $\begin{array}{l}\text { Ruksha, } \\
\text { Laghu }\end{array}$ & Ushna & Katu & $\begin{array}{l}\text { Kaphapitta Shamaka, } \\
\text { Shothahara, } \\
\text { Krimighna, } \\
\text { Vishaghna }\end{array}$ & $\begin{array}{l}\text { Kushtaghna, } \\
\text { Vishaghna, } \\
\text { Kandugna, } \\
\text { Tiktaskanda, } \\
\text { Shirovirechana } \\
\text { Lekhaniya Varga }\end{array}$ \\
\hline 3 & Ativisha $^{5}$ & $\begin{array}{l}\text { Tikta, } \\
\text { Katu }\end{array}$ & $\begin{array}{l}\text { Laghu, } \\
\text { Ruksha }\end{array}$ & Ushna & Katu & $\begin{array}{l}\text { Kaphapitta Shamaka, } \\
\text { Krimighna, Vishaghna, } \\
\text { Jwarahara }\end{array}$ & $\begin{array}{l}\text { Lekhaniya, Ar- } \\
\text { shoghna }\end{array}$ \\
\hline 4 & Abhaya $^{6}$ & $\begin{array}{l}\text { Kashaya,Amla, } \\
\text { Madhura,Katu, } \\
\text { Tikta }\end{array}$ & $\begin{array}{l}\text { Laghu } \\
\text { Ruksha }\end{array}$ & Ushna & Madhura & $\begin{array}{l}\text { Tridoshashamaka } \\
\text { Rasayana, Anulomana, } \\
\text { Krimighna }\end{array}$ & $\begin{array}{l}\text { Triphala } \\
\text { Amalakyadi } \\
\text { Parushakadi } \\
\text { Arshoghna, }\end{array}$ \\
\hline
\end{tabular}




\begin{tabular}{|c|c|c|c|c|c|c|c|}
\hline & & & & & & $\begin{array}{l}\text { Kandughna, Shothahara, } \\
\text { Deepana } \\
\text { Pachana }\end{array}$ & $\begin{array}{l}\text { Kushtaghna, } \\
\text { Jwarahara } \\
\text { Prajyasthapana } \\
\text { Kasaghna }\end{array}$ \\
\hline 5 & $A b d h a^{7}$ & $\begin{array}{l}\text { Tikta, } \\
\text { Katu } \\
\text { Kashaya }\end{array}$ & $\begin{array}{l}\text { Laghu, } \\
\text { Ruksha }\end{array}$ & Sheeta & Katu & $\begin{array}{l}\text { Pittakapha Shamaka } \\
\text { Krimighna } \\
\text { Jwarahara } \\
\text { Dipana } \\
\text { Pachana }\end{array}$ & $\begin{array}{l}\text { Tripthighna } \\
\text { Varga } \\
\text { Trishnanigra- } \\
\text { hana } \\
\text { Lekhaniya } \\
\text { Kandughna } \\
\text { Sthanyashodhaka }\end{array}$ \\
\hline 6 & Harenuka $^{8}$ & $\begin{array}{l}\text { Katu } \\
\text { Tikta }\end{array}$ & $\begin{array}{l}\text { Laghu, } \\
\text { Ruksha }\end{array}$ & Ushna & Katu & $\begin{array}{l}\text { Vatakapha Shamaka } \\
\text { Shothahara } \\
\text { Krimighna } \\
\text { Kushtaghna } \\
\text { Kandughna }\end{array}$ & $\begin{array}{l}\text { Vishaghna } \\
\text { Krimighna }\end{array}$ \\
\hline 7 & $E l a^{9}$ & $\begin{array}{l}\text { Katu, } \\
\text { Madhura }\end{array}$ & $\begin{array}{l}\text { Laghu, } \\
\text { Ruksha }\end{array}$ & Sheeta & Madhura & $\begin{array}{l}\text { Tridoshahara } \\
\text { Rochana } \\
\text { Hridhya } \\
\text { Dipana } \\
\text { Shwasahara } \\
\text { Kasahara }\end{array}$ & $\begin{array}{l}\text { Shwasahara } \\
\text { Angamarda- } \\
\text { Prashamana } \\
\text { Katuskanda }\end{array}$ \\
\hline 8 & Vakra $^{10}$ & $\begin{array}{l}\text { Tikta } \\
\text { Katu, } \\
\text { Kashya }\end{array}$ & $\begin{array}{l}\text { Laghu, } \\
\text { Snigdha }\end{array}$ & Ushna & Katu & $\begin{array}{l}\text { Kaphavatashamaka } \\
\text { Vedanasthapana } \\
\text { Vranaropana } \\
\text { Vishaghna }\end{array}$ & $\begin{array}{l}\text { Sheetaprasha- } \\
\text { mana } \\
\text { Tiktaskanda }\end{array}$ \\
\hline 9 & Kushta ${ }^{11}$ & $\begin{array}{l}\text { Tikta, } \\
\text { Katu } \\
\text { Madhura }\end{array}$ & $\begin{array}{l}\text { Laghu, } \\
\text { Ruksha } \\
\text { Tikshna }\end{array}$ & Ushna & Katu & $\begin{array}{l}\text { Kaphavatashamaka } \\
\text { Jantughna } \\
\text { Vedanasthapana } \\
\text { Kushtsghna }\end{array}$ & $\begin{array}{l}\text { Lekhaniya } \\
\text { Shukrashodhana } \\
\text { Asthapanopaga }\end{array}$ \\
\hline 10 & Priyang $u^{12}$ & $\begin{array}{l}\text { Tikta, } \\
\text { Kashaya, } \\
\text { Madhura }\end{array}$ & $\begin{array}{l}\text { Guru, } \\
\text { Ruksha }\end{array}$ & Sheeta & Katu & $\begin{array}{l}\text { Tridosha Shamaka, } \\
\text { Dahaprashamana, } \\
\text { danasthapana, } \\
\text { gandhanashana }\end{array}$ & $\begin{array}{l}\text { Mutravira- } \\
\text { janeeya, } \\
\text { Pureesha- } \\
\text { Sangrahaneeya }\end{array}$ \\
\hline
\end{tabular}

Fig. 1: Analysis of Rasa

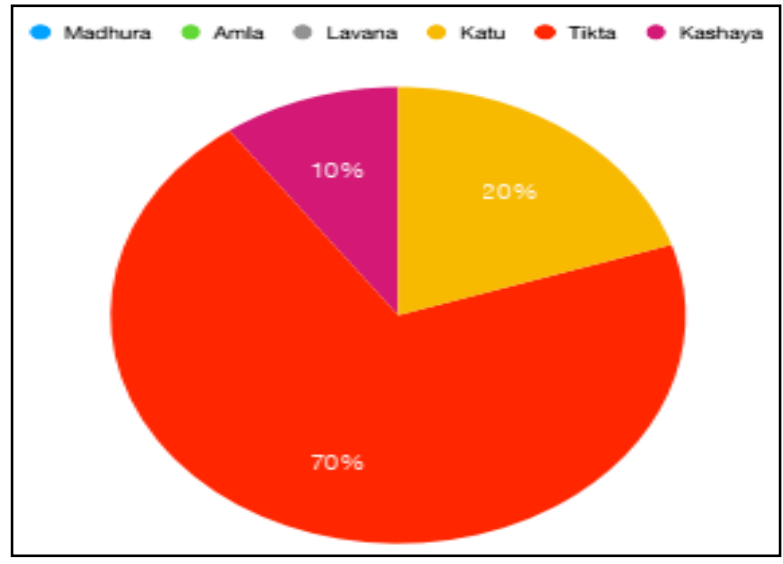

Fig. 3: Analysis of Veerya
Fig. 2: Analysis of Guna

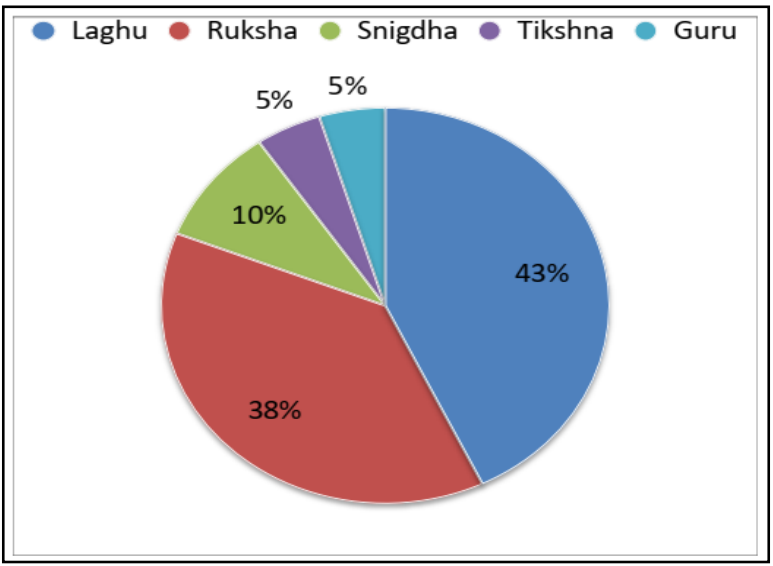

Fig. 4: Analysis of Vipaka 

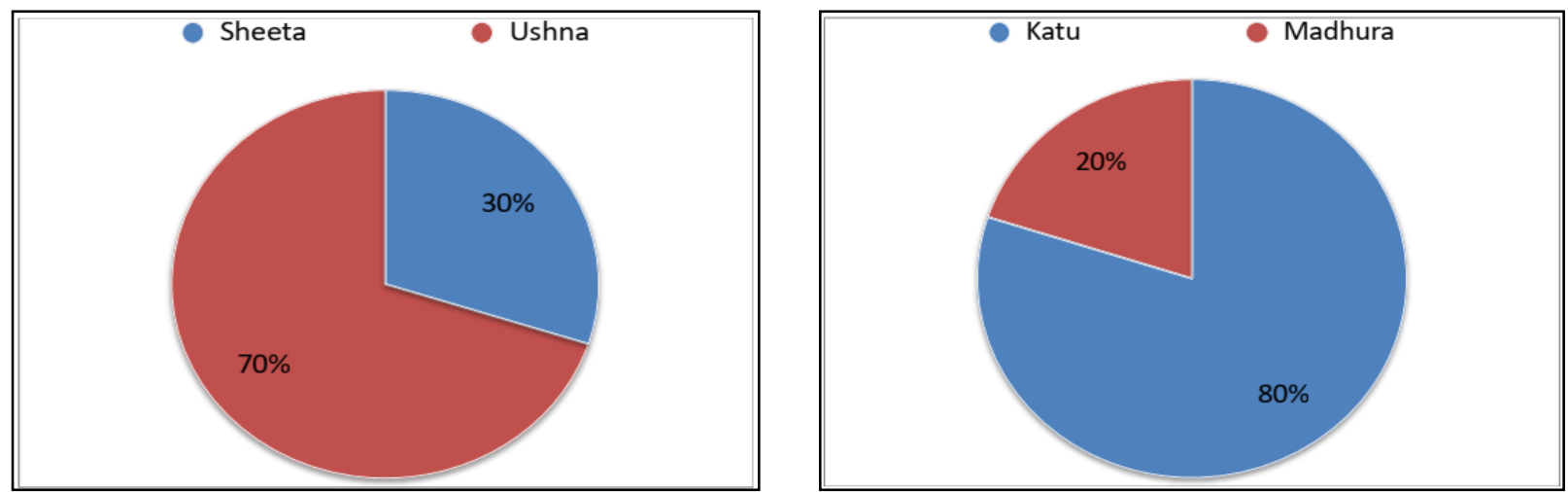

Table 3: Phyto-constituents of ingredients of Laaksha haridradi dhoopa

\begin{tabular}{|c|c|c|}
\hline S. No & Drug name & Phyto- constituents \\
\hline 1 & Laksha ${ }^{13}$ & $\begin{array}{l}\text { Stick lac contains } 70-80 \% \text { of resin, sugars, proteins, colouring matter }(1-2 \%) \text {, wax (4- 6\%), extrane- } \\
\text { ous matter }(8-12 \%) \text { and volatile oil in traces. } \\
\text { Lac resin consists of inter-esters of hydroxyl fatty acid derivatives. } \\
\text { Aleuritic acid is the main constituent } 35 \% \text { of resin, while shellolic acid and its isomers along with } \\
\text { kerrolic acid } \quad \text { and } \quad \text { butolic } \\
\text { persent to a small extent. }\end{array}$ \\
\hline 2 & Haridra ${ }^{14}$ & $\begin{array}{l}\text { Rhizome contains about } 5 \% \text { of volatile oil, resin, abundant zingiberaceous starch grains and yellow } \\
\text { coloring substances known as curcuminoids. Chief component of curcuminoids is known as curcu- } \\
\text { min }(50-60 \%)\end{array}$ \\
\hline 3 & Ativisha ${ }^{15}$ & $\begin{array}{l}\text { The root yield } 0.79 \% \text { of total alkaloids, of which atisin is } 0.4 \% \text {. The plant possesses potent immune } \\
\text { stimulant property, }\end{array}$ \\
\hline 4 & Abhaya ${ }^{16}$ & Moisture- $10 \%$, Tannin $-25-32 \%$, Water insoluble matter- $40-50 \%$ \\
\hline 5 & $A b d h a^{17}$ & $\begin{array}{l}\text { The tuber is rich in } \mathrm{Cu}, \mathrm{Fe}, \mathrm{Mg} \text { and } \mathrm{Ni} \text {. Beta- sitosterol, is isolated from tuber, exhibits significant } \\
\text { anti-inflammatory activity against carrageenan and cotton pellet induced edema in rats. }\end{array}$ \\
\hline 6 & Harenuka ${ }^{18}$ & $\begin{array}{l}\text { The seeds contain p-hydroxybenzoic acid, 5- oxyisophthalic acid, glucose and the triterpene, vitex- } \\
\text { triterpene. }\end{array}$ \\
\hline 7 & $E a^{19}$ & $\begin{array}{l}\text { Seeds contain volatile oil } 2-8 \% \text {. The active constituent of volatile oil is cineole. Aromatic compounds } \\
\text { present are terpinyl acetate, terpineol, borneol, terpinene etc. }\end{array}$ \\
\hline 8 & Vakra $^{20}$ & $\begin{array}{l}\text { It contains pale brown to amber colored oil about } 1 \% \text { and alkaloids chatinine and valerine. Volatile } \\
\text { oil contains borneol formate, borneol acetate, camphene etc. Valepotriates or valtrate responsible for } \\
\text { therapeutic activity of the drug. }\end{array}$ \\
\hline 9 & Kushta ${ }^{21}$ & $\begin{array}{l}\text { Root contains about } 6 \% \text { resinoids, } 1.5 \% \text { of essential oil, } 18 \% \text { of inulin, } 0.5 \% \text { of an alkaloid, fixed } \\
\text { oil, traces of tannins and sugar. The alkaloid is known as saussurine, Kushin. }\end{array}$ \\
\hline 10 & Priyang $u^{22}$ & $\begin{array}{l}\text { The seeds and leaves contain calliterpenone and its monoacetate; the former contain fatty acids, beta- } \\
\text { sitosterol and its beta- D- glucose. }\end{array}$ \\
\hline
\end{tabular}

Method of Preparation: In the reference Acharya Sushruta mentioned that the above listed ten drugs should be added to fire and the dhuma (fume) comes from this will purify the polluted air.

\section{DISCUSSION}

Dhoopana karma is one of the anti-microbial procedures used in Indian tradition in different forms like Homa, Yajna etc., to purify the air.
In our classics Acharyas mentioned many of the combinations without proper nomenclature. Laaskha Haridradi yoga is one such yoga mentioned Sushruta Samhita Kalpasthana for the purification of the polluted air.

In this yoga the majority of the drugs are having Tikta, Katu Rasa, Ushna Veerya and Katu Vipaka. Drugs like Haridra, Ativisha, and Vakra are having anti poisonous effect. The drugs like Haridra, Ativisha, Kushta, Tagara, are having a potential antimicrobial activity. 
The drugs like Haridra, Ela and Tagara are having volatile aromatic oil which helps to remove the bad odour and gives freshness to air.

\section{CONCLUSION}

In Indian tradition from time immemorial the Dhupana process is well established and it is practiced regularly for different purposes. Laaskha Haridradi yoga is one such yoga mentioned Sushruta Samhita Kalpasthana for the purification of the polluted air. Here ten drugs were mentioned out of which many drugs are having good antimicrobial and anti-poisonous activities. Hence a detailed antimicrobial study can be done to elaborate the role and efficacy of Laaksha Haridradi yoga.

\section{REFERENCES}

1. Charaka Samhita Vimana Sthana; Vyadhitrirupiya Adhyaya: Chapter 7 Verse 9, Charaka Samhita, Vidyotini Hindi Commentary by Kashinath Shastri \& Gorakhnath Chaturvedi, Part-I, Published By Chaukhambha Bharati Academy, Varanasi, India, Reprint Year; 2009; Pp-837; P258

2. Acharya JT, Susrutha Samhitha Of Susrutha Nibandha Samgraha Commentary of Sri Dalhana Acharya And Nyaya Chandrikapanjika Of Sri Gayadasa Acharya. Kalpasthana; Jangama Vishavijnaniya Kalpa Adhyaya: 3/16-17.Varanasi. Chaukambha Sanskrit Sansthan; 2009; P 568-569

3. Pandey G. Dravyaguna Vijnana. Reprint. Varanasi: Chowkhamba Krishnadasa Academy;2004. Vol II. Pp822.P-388

4. Sharma, Priyavrit Dravyaguna Vijnana, Vol II, Reprint 2005, Varanasi: Chaukambha Bharati Academy Varanasi. Pp -873. P-162

5. Sharma, Priyavrit Dravyaguna Vijnana, Vol II, Reprint 2005, Varanasi: Chaukambha Bharati Academy Pp 873. P-355

6. Sharma, Priyavrit Dravyaguna Vijnana, Vol II, Reprint 2005, Varanasi: Chaukambha Bharati Academy Pp 873. P- 753

7. Sharma, Priyavrit Dravyaguna Vijnana, Vol II, Reprint 2005, Varanasi: Chaukambha Bharati Academy Pp 873. P- 370

8. Sharma, Priyavrit Dravyaguna Vijnana, Vol II, Reprint 2005, Varanasi: Chaukambha Bharati Academy Pp 873. P- 66

9. Sharma, Priyavrit Dravyaguna Vijnana, Vol II, Reprint 2005, Varanasi: Chaukambha Bharati Academy Pp 873. P- 716
10. Sharma, Priyavrit Dravyaguna Vijnana, Vol II, Reprint 2005, Varanasi: Chaukambha Bharati Academy Pp 873. P- 64

11. Sharma, Priyavrit Dravyaguna Vijnana, Vol II, Reprint 2005, Varanasi: Chaukambha Bharati Academy Pp 873. P- 572

12. Sharma, Priyavrit Dravyaguna Vijnana, Vol II, Reprint 2005, Varanasi: Chaukambha Bharati Academy Pp 873. P- 781

13. Kokate C K, Purohit AP, Gokhale SB, Pharmacognosy. 39th Edition 2007, Pune, Nirali Prekashana. Pp-606. P429

14. Kokate C K, Purohit AP, Gokhale SB, Pharmacognosy. 39th Edition 2007, Pune, Nirali Prekashana. Pp-606. P402

15. Khare C. P, Indian Medicinal Plant, An Illustrated Dictionary, 2007, Newyork, Springer Science + Business Media LLC. Pp -812. P-

16. Kokate C K, Purohit AP, Gokhale SB, Pharmacognosy. 39th Edition 2007, Pune, Nirali Prekashan. Pp-606. P258.

17. Khare C. P. Indian Medicinal Plant, An Illustrated Dictionary, 2007, Newyork, Springer Science + Business Media LLC. Pp -812. P- 195

18. Khare C. P. Indian Medicinal Plant, An Illustrated Dictionary, 2007, Newyork, Springer Science + Business Media LLC. Pp -812. P- 711

19. Kokate C K, Purohit AP, Gokhale SB, Pharmacognosy. 39th Edition Pune, 2007, Pune Nirali Prekashan. Pp606. P- 329.

20. Kokate C K, Purohit AP, Gokhale SB, Pharmacognosy. 39th Edition 2007, Pune, Nirali Prekashan. Pp-606. P382.

21. Kokate C K, Purohit AP, Gokhale SB, Pharmacognosy. 39th Edition 2007, Pune, Nirali Prekashan. Pp-606. P378.

22. Khare C.P. Indian Medicinal Plant, An Illustrated Dictionary, 2007, Newyork, Springer Science + Business Media LLC. Pp-812. P- 711.

\section{Source of Support: Nil Conflict of Interest: None Declared}

How to cite this URL: Ravikrishna S. \& Sreejith K: A Review on Laaksha Haridradi Dhoopa. International Ayurvedic Medical Journal \{online\} 2020 \{cited July, 2020\} Available from:

http://www.iamj.in/posts/images/upload/2422 2426.pdf 